\title{
Aberrant promoter methylation of T-cadherin in sera is associated with a poor prognosis in oral squamous cell carcinoma
}

\author{
Qiuju WANG ${ }^{1, *}$, Yan $\mathrm{CHEN}^{1, *}$, Yibo $\mathrm{CHEN}^{1}$, Jian JIANG ${ }^{1}$, Xiaoyu SONG ${ }^{1}$, Li ZHANG ${ }^{1}$, Qiao HE ${ }^{1}$, Bo YE ${ }^{1}$, Lichun WU ${ }^{1}$, Rui WU ${ }^{1}$, Qin LAI ${ }^{1}$, \\ Dongsheng WANG ${ }^{1, *}$, Yanzhen $\mathrm{ZHAO}^{2, *}$ \\ ${ }^{1}$ Sichuan Cancer Hospital and Institute, University of Electronic Science and Technology of China, Chengdu, China; ${ }^{2}$ Department of Clinical \\ Laboratory, The Third Affiliated Hospital of Guangzhou Medical University, Liwan, Guangzhou, China
}

*Correspondence: zhyzh5566@163.com; 13990820268@163.com

${ }^{*}$ Contributed equally to this work.

Received November 10, 2020 / Accepted January 4, 2021

\begin{abstract}
T-cadherin functions as a suppressor gene, which is frequently inactivated by aberrant promoter methylation in several human cancers, but its methylation status in oral squamous cell carcinoma (OSCC) has been scarcely studied. Thus this study aimed at exploring the clinical significance and prognostic value of T-cadherin methylation in sera of patients with OSCC. Methylation-specific PCR (MSP) and bisulfate sequencing PCR (BSP) was performed to examine the methylation status of $\mathrm{T}$-cadherin. Then, the associations between methylation status of T-cadherin and various clinicopathological variables or patient survival were investigated in 202 patients with OSCC and 68 controls. T-cadherin methylation was detected in 62 out of $202(30.7 \%)$ patients with OSCC, and the methylation status of T-cadherin in corresponding tissues was confirmed by BSP. Methylation of T-cadherin was significantly associated with advanced tumor T-stage $(\mathrm{p}<0.001)$ and $\mathrm{N}$-stage $(\mathrm{p}=0.003)$, positive lymphatic metastasis $(\mathrm{p}=0.004)$ and tumor recurrence $(\mathrm{p}=0.001)$. In addition, patients with methylation of T-cadherin had worse overall survival $(\mathrm{p}=0.018)$ and progression-free survival $(\mathrm{p}<0.001)$ than patients without, and methylation of T-cadherin in sera was an independent prognostic factor for worse overall survival (HR: 3.626, 95\% CI: 1.112-9.624, $\mathrm{p}=0.007$ ) and progression-free survival (HR: 4.201, 95\% CI: $1.562-10.038, \mathrm{p}<0.001$ ) of patients with OSCC. These results demonstrated that methylation of T-cadherin was frequently detected in sera of patients with OSCC, which was associated with risk factors of poor outcomes, and may act as a potential independent prognostic marker for patients with OSCC.
\end{abstract}

Key words: T-cadherin, methylation, serum, prognosis, OSCC

Oral squamous cell carcinoma (OSCC) is the most common oral and maxillofacial malignancy, with an estimated 350,000 new cases and 170,000 deaths in 2018 worldwide [1]. Surgery, chemotherapy, radiotherapy, or combinations of these are the main treatment modalities for OSCC patients [2]. Nevertheless, the survival rate of OSCC patients remains low due to loco-regional recurrence [3]. Targeted therapy against epidermal growth factor receptors and checkpoint inhibitor-based immunotherapy have recently been used for the treatment of OSCC, such as cetuximab, pembrolizumab, and nivolumab [4-6]. However, there is a limited number of targeted therapies for OSCC patients, and only a small fraction of patients who received checkpoint inhibitor-based immunotherapy responded to it [7]. This means that there are limited treatment options for a large proportion of OSCC patients. Therefore, searching for effective biomarkers and novel therapeutic targets is of great importance for these patients.
Epigenetic modifications are important mechanisms in carcinogenesis, and the epigenetic change that has been studied the most in relation to OSCC is DNA hypermethylation [8]. A recent study has shown that the silencing of tumor suppressor genes through hypermethylation of the $\mathrm{CpG}$ islands on the gene promoters is associated with the formation and progression of OSCC [9]. DNA methylation can be detected not only in tumor tissues but also in serum [10], and detection of DNA methylation in serum is of great value in the early detection, treatment, and prognosis evaluation of patients with cancer [11]. T-cadherin, a member of the cadherin family, functions as a tumor suppressor gene in various tumors [12-13]. Our previous study found that T-cadherin was lower in OSCC tissues and that can inhibit proliferation of OSCC cells through the PI3K/Akt/mTOR pathway [14]. This study also found that the methylation of T-cadherin in sera has been detected in several other tumors 
[15-16], but the methylation status of T-cadherin in the sera of OSCC patients remains unclear.

The aim of the study is to investigate the methylation status of T-cadherin in the sera of OSCC patients and correlated it with various clinicopathological characteristics and patient outcomes to determine its clinical significance.

\section{Patients and methods}

Patients, sera, and tissue samples. PASS 15.0 software was used for power analysis and sample size calculating and at last, a total of 202 patients who underwent surgical resection for primary OSCC at Sichuan Cancer Hospital \& Institute between January 2011 and August 2019 were enrolled in this study. The inclusion criteria were as follows: patients with pathologically diagnosed OSCC, patients were followed up and willing to take part in the study, provided written informed consent; the exclusion criteria were patients received anti-cancer treatment before, patients had prior malignancy, patients had metastasis at diagnosis, and patients had other systemic diseases [17]. All the patients with OSCC were staged according to the clinical tumor-node-metastasis classification system of the American Joint Committee on Cancer/Union for International Cancer Control guideline. Patients in the same stage all received the same standard treatment. Peripheral blood samples $(10 \mathrm{ml})$ were collected within one week before surgery and centrifuged at $1800 \mathrm{~g}$ for $10 \mathrm{~min}$ [18]. Sera were collected and stored at $-80^{\circ} \mathrm{C}$ until analysis. Among the 202 patients, 8 specimens of OSCC cancerous tissues and adjacent normal tissues were collected. The tissues were preserved at temperature of $-80^{\circ} \mathrm{C}$ before utilization. Written informed consent was obtained from each patient and healthy control. The study was conducted according to the Declaration of Helsinki and approved by the ethics committee of Sichuan Cancer Hospital and Institute.

DNA extraction, bisulfite conversion, and methylationspecific PCR (MSP). DNA was extracted from sera of patients according to the instructions of the QIAmp DNA Blood Mini Kit (Tiangen Biotech, China) [18], and the isolated DNA was modified by bisulfite using EZ DNA Methylation-Gold kit (Zymo Research, USA). MSP was used to detect the methylation status of T-cadherin in sera. The PCR conditions were as follows: pre-denaturation at $95^{\circ} \mathrm{C}$ for $5 \mathrm{~min}$, denaturation at $95^{\circ} \mathrm{C}$ for $30 \mathrm{~s}$, annealing at $60^{\circ} \mathrm{C}(\mathrm{U}) / 70^{\circ} \mathrm{C}(\mathrm{M})$ for $1 \mathrm{~min}$, extension at $72^{\circ} \mathrm{C}$ for $1 \mathrm{~min}$, and a final extension at $72^{\circ} \mathrm{C}$ for 5 min. Primer sequences were shown in Table 1, the length of the amplified sequence was $242 \mathrm{bp}$, PCR products were separated on $2 \%$ agarose gel and the images were processed by quantity one system.

Bisulfite sequencing PCR (BSP). The Universal DNA Purification Kit (Tiangen Biotech, China) was used to extract and purify DNA from tissue specimens according to the manufacturer's protocol [19]. The CpG island was predicted with Methprimer and the primer (Table 1) was designed according to the sequence of the $\mathrm{CpG}$ island. The length of the amplified fragment was $241 \mathrm{bp}$. The conditions of reaction were as follows: $94^{\circ} \mathrm{C}$ for 3 minutes, $\left(94^{\circ} \mathrm{C}\right.$ for $30 \mathrm{~s}, 55^{\circ} \mathrm{C}$ for $30 \mathrm{~s}, 72^{\circ} \mathrm{C}$ for $15 \mathrm{~s}$ ) through 30 cycles and $72^{\circ} \mathrm{C}$ for $10 \mathrm{~min}$. The PCR products were purified and cloned into the pMD19-T vector (TAKARA, China), 10 of the ligated products were introduced into the DH5a cell line. All clones were sequenced (Sangon, China) and analyzed with the UltraEdit Professional Text/Hex Editor.

Statistical analysis. Controls were obtained from healthy physical examination patients matched by age, sex, and socioeconomic status, besides, OSCC patients and controls were propensity score-matched. Fisher's exact test was used to compare the difference of T-cadherin methylation status between OSCC patients and controls. Chi-square test was used to evaluate the correlations between the methylation status of T-cadherin and clinicopathological variables. Kaplan-Meier analysis was used to plot the survival curves and the log-rank test was used to determine the significance. The prognostic value of the methylation status of T-cadherin in OSCC was evaluated by univariate and multivariate cox proportional hazard model. The statistical analyses were carried out by using SPSS 16.0 software and the $p$-value $<0.05$ was considered statistically significant.

\section{Results}

Main clinical and pathological characteristics of patients with OSCC. A total of 202 patients were enrolled in this study. The main clinical and pathological characteristics of patients with OSCC are shown in Table 2. 151 (74.75\%) patients were males and $51(25.25 \%)$ patients were females. $66(32.67 \%)$ patients were lymphatic metastasis positive and 29 (14.36\%) were surgical margins positive, and 79 (39.11\%) patients relapsed at the last follow-up date. We calculated progression-free survival (PFS) from the OSCC patients' date of surgery to the date of clinicopathological evidence of cancer recurrence, and the overall survival (OS) was calculated from the OSCC patients' date of surgery to the date of death or to the last contact date.

The methylation status of T-cadherin in sera and tissues of OSCC patients and controls. The methylation status of T-cadherin in sera was detected by methylation-specific PCR (MSP). The methylation of T-cadherin in sera was detected

Table 1. Primer sequences for MSP and BSP.

\begin{tabular}{ll}
\hline T-cadherin (M) & F: 5'-TCGCGGGGTTCGTTTTTCGC-3' \\
& R: 5'-GACGTTTTCATTCATACACGCG-3' \\
T-cadherin (U) & F: 5'-TTGTGGGGTTGTTTTTTGT-3' \\
& R: 5'-AACTTTTCATTCATACACACA-3' \\
BSP & F: 5'-ATTTTTTGGAAAAGTGGAATTAGTT-3' \\
& R: 5'-CCAAATAAATCAACAACAACATCAC-3' \\
\hline
\end{tabular}

Abbreviations: F-forward; R-reverse; U-unmethylated T-cadherin promoter; M-methylated T-cadherin promoter; BSP-bisulfite sequencing PCR 
Table 2. The clinical and pathological characteristics of patients with OSCC.

\begin{tabular}{lll}
\hline Variables & Cases $(\mathrm{n}=202)$ & $\%$ \\
\hline Gender & & \\
Male & 151 & 74.75 \\
Female & 51 & 25.25 \\
Age & & \\
$\quad \leq 60$ & 92 & 45.54 \\
$>60$ & 110 & 54.46 \\
$\mathrm{~T}_{\text {classification }}$ & & \\
$\mathrm{T}_{1-2}$ & 90 & 44.55 \\
$\mathrm{~T}_{3^{-4}}$ & 112 & 55.45 \\
$\mathrm{~N}_{\text {classification }}$ & & \\
$\quad \mathrm{N}_{0-1}$ & 128 & 63.37 \\
$\quad \mathrm{~N}_{2-3}$ & 74 & 36.63 \\
Lymphatic metastasis & & \\
No & 136 & 67.33 \\
Yes & 66 & 32.67 \\
Margins & & \\
Negative & 173 & 85.64 \\
Positive & 29 & 14.36 \\
Recurrence & & 60.89 \\
No & 123 & 39.11 \\
Yes & 79 & \\
\hline
\end{tabular}

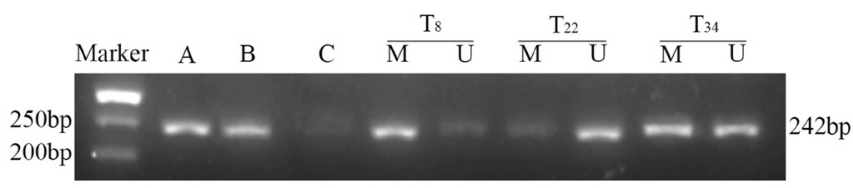

Figure 1. Representative MSP results of T-cadherin methylation in sera of OSCC patients. A) positive methylated control in vitro; B) positive unmethylated control; C) water blank. T8 and T34 exhibited methylated T-cadherin, T22 exhibited unmethylated T-cadherin.

in $30.7 \%(62 / 202)$ of OSCC patients, and a representative MSP result is shown in Figure 1, however, no methylation was detected in the controls. The difference between the 2 groups was statistically significant $(\mathrm{p}<0.001)$.

In order to explore the origin of T-cadherin methylation among the 62 patients with T-cadherin methylation detected in sera, we further conducted bisulfite sequencing (BSP) to determine the $\mathrm{CpG}$ island methylation status of T-cadherin in 8 pairs of corresponding OSCC tissues and adjacent normal ones. Using the Methprimer website [19], we identified a C-phosphate- $\mathrm{G}(\mathrm{CpG})$ island in the promoter region of T-cadherin (Figure 2A). The results demonstrated that the methylation percentage of T-cadherin was higher in OSCC tissues than adjacent normal ones (Figures 2B, 2C), which is consistent with the MSP results in sera.
Table 3. The correlation between T-cadherin methylation in serum and clinicopathological features of patients with OSCC.

\begin{tabular}{|c|c|c|c|c|}
\hline Variables & $\mathbf{n}$ & M (\%) & U (\%) & p-value ${ }^{a}$ \\
\hline Gender & & & & 0.351 \\
\hline Male & 151 & $49(32.5)$ & $102(67.5)$ & \\
\hline Female & 51 & $13(25.5)$ & $38(74.5)$ & \\
\hline Age & & & & 0.321 \\
\hline$\leq 60$ & 92 & $25(27.2)$ & $67(72.8)$ & \\
\hline$>60$ & 110 & $37(33.6)$ & $73(66.4)$ & \\
\hline $\mathrm{T}$ classification & & & & $<0.001^{*}$ \\
\hline $\mathrm{T}_{1}$ or $\mathrm{T}_{2}$ & 90 & $14(15.6)$ & $76(84.4)$ & \\
\hline $\mathrm{T}_{3}$ or $\mathrm{T}_{4}$ & 112 & $48(42.9)$ & $64(57.1)$ & \\
\hline $\mathrm{N}$ classification & & & & $0.003^{*}$ \\
\hline $\mathrm{N}_{0}$ or $\mathrm{N}_{1}$ & 128 & $30(23.4)$ & $98(76.6)$ & \\
\hline $\mathrm{N}_{2}$ or $\mathrm{N}_{3}$ & 74 & $32(43.2)$ & $42(56.8)$ & \\
\hline Lymphatic metastasis & & & & $0.004^{*}$ \\
\hline No & 136 & $33(24.3)$ & $103(75.7)$ & \\
\hline Yes & 66 & $29(43.9)$ & $37(56.1)$ & \\
\hline Margins & & & & 0.633 \\
\hline Negative & 173 & $52(30.1)$ & $121(69.9)$ & \\
\hline Positive & 29 & $10(34.5)$ & $19(65.5)$ & \\
\hline Recurrence & & & & $0.001^{*}$ \\
\hline No & 123 & $27(21.2)$ & $96(78.0)$ & \\
\hline Yes & 79 & $35(44.3)$ & $44(55.7)$ & \\
\hline Total & 202 & $62(30.7)$ & $140(69.3)$ & \\
\hline
\end{tabular}

Notes: ${ }^{a} \chi^{2}$ test was used to evaluate the correlations between the methylation status of T-cadherin and clinicopathological variables; ${ }^{\star} \mathrm{p}$-value $<0.05$ was considered statistically significant. Abbreviations: U-unmethylated

$\mathrm{T}$-cadherin promoter; M-methylated T-cadherin promoter

The association between methylation status of T-cadherin and various clinicopathological variables of OSCC. The correlation between methylation status of $\mathrm{T}$-cadherin and various clinicopathological variables of OSCC is shown in Table 3. The results suggest that the methylation of T-cadherin was significantly correlated with advanced $\mathrm{T}$ classification $(\mathrm{p}<0.001)$, advanced $\mathrm{N}$ classification $(\mathrm{p}=0.003)$, positive lymphatic metastasis $(\mathrm{p}=0.004)$, and tumor recurrence $(\mathrm{p}=0.001)$. However, no significant correlation was found between methylation of T-cadherin and gender, age, or margins.

The prognostic value of $\mathrm{T}$-cadherin methylation in sera of OSCC patients. As shown in Figure 3, the KaplanMeier analysis results suggest that patients with methylated T-cadherin in sera had shorter overall survival $(p=0.018$, Figure $3 \mathrm{~A})$ and progression-free survival $\quad(\mathrm{p}<0.001$, Figure $3 \mathrm{~B}$ ) than those with unmethylated T-cadherin. Univariate analysis showed that $\mathrm{T}$ stage, $\mathrm{N}$ stage, surgical margin status, and T-cadherin methylation were associated with OS (Table 4) and PFS (Table 5) of patients with OSCC. Multivariate analysis suggested that methylation of T-cadherin is an independent prognostic factor for OS (HR: 3.626, 95\% CI: 1.112-9.624, $\mathrm{p}=0.007$, Table 4) and PFS (HR: 4.201, 95\% CI: $1.562-10.038, \mathrm{p}<0.001$, Table 5) of patients with OSCC. 


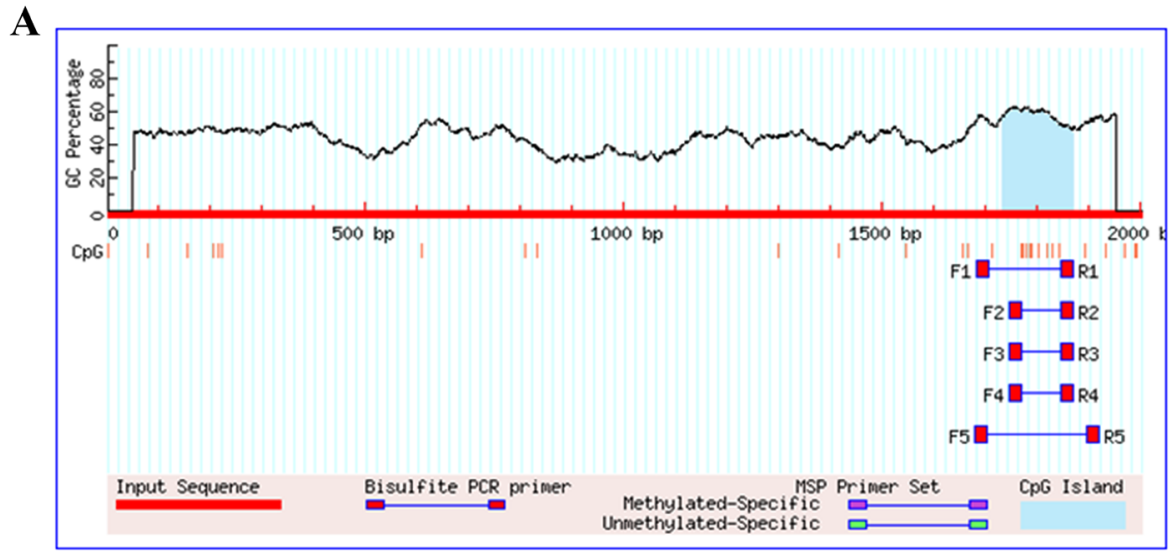

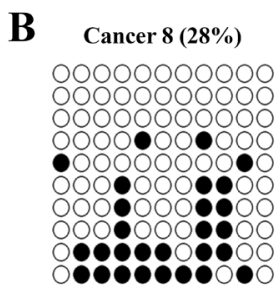

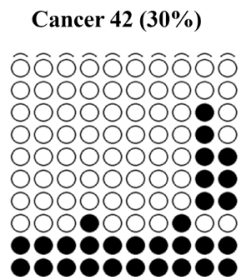

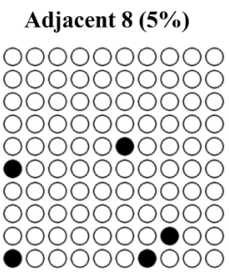

Adjacent $42(18 \%)$

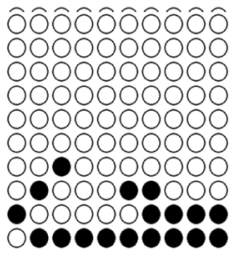

C

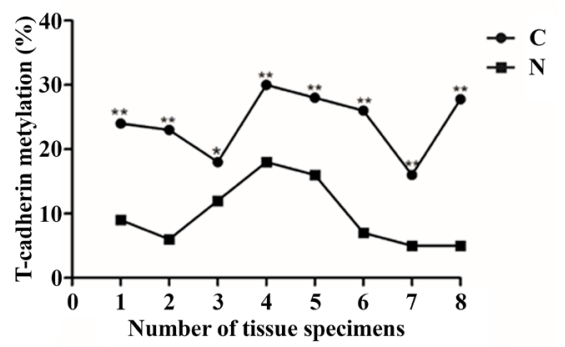

Figure 2. CpG island methylation level of T-cadherin promoter measured by the BSP assay. A) Analysis of CpG island enrichment in the T-cadherin promoter region. B) Representative images and corresponding statistical plots are presented. Black solid circles represent methylated CpG sites, and white hollow circles represent unmethylated CpG sites. C) The methylation percentage of T-cadherin in 8 pairs of OSCC tissues and adjacent normal tissues. ${ }^{*} \mathbf{p}<0.05$

Table 4. Univariate and multivariate analysis of the prognostic value of T-cadherin methylation regarding OS in patients with OSCC.

\begin{tabular}{|c|c|c|c|c|c|c|}
\hline \multirow{2}{*}{ Variables } & \multicolumn{3}{|c|}{ Univariate analysis } & \multicolumn{3}{|c|}{ Multivariate analysis } \\
\hline & HR & 95\% CI & p-value & HR & 95\% CI & p-value \\
\hline Gender & 1.782 & $0.968-4.132$ & 0.158 & & & \\
\hline Age & 1.258 & $0.882-2.736$ & 0.266 & & & \\
\hline T- classification & 2.462 & $1.434-5.864$ & $0.012^{*}$ & 2.269 & $1.368-5.362$ & $0.026^{*}$ \\
\hline $\mathrm{N}$ - classification & 2.732 & $1.532-6.038$ & $0.008^{\star}$ & 2.468 & $1.442-5.658$ & $0.013^{*}$ \\
\hline Lymphatic metastasis & 1.296 & $0.796-3.126$ & 0.625 & & & \\
\hline Margins & 3.022 & $1.326-9.582$ & $<0.001^{*}$ & 2.966 & $1.288-8.964$ & $0.026^{*}$ \\
\hline Recurrence & 1.529 & $0.838-3.276$ & 0.325 & & & \\
\hline T-cadherin methylation & 3.854 & $1.065-10.682$ & $0.002^{*}$ & 3.626 & $1.112-9.624$ & $0.007^{\star}$ \\
\hline
\end{tabular}

Note: ${ }^{*}$-value $<0.05$ was considered statistically significant

\section{Discussion}

Promoter methylation is the most common and representative epigenetic change and is the main mechanism of tumor suppressor gene inactivation [20]. Studies have found that tumor suppressor gene methylation can be detected not only in tumor tissues but also in various body fluids including sera [21]. A large number of DNA fragments can be detected in the sera of tumors, including OSCC [22], so the methylation of circulating tumor-related DNA might be able to be used as 
Table 5. Univariate and multivariate analysis of the prognostic value of T-cadherin methylation regarding PFS in patients with OSCC.

\begin{tabular}{|c|c|c|c|c|c|c|}
\hline \multirow{2}{*}{ Variables } & \multicolumn{2}{|c|}{ Univariate analysis } & \multirow[b]{2}{*}{ p-values } & \multicolumn{3}{|c|}{ Multivariate analysis } \\
\hline & HR & $95 \% \mathrm{CI}$ & & HR & $95 \% \mathrm{CI}$ & p-values \\
\hline Gender & 1.852 & $0.832-3.966$ & 0.145 & & & \\
\hline Age & 1.466 & $0.758-3.762$ & 0.322 & & & \\
\hline T-classification & 2.682 & $1.568-5.968$ & $0.008^{*}$ & 2.468 & $1.268-4.968$ & $0.01^{\star}$ \\
\hline N-classification & 2.864 & $1.682-6.012$ & $0.004^{*}$ & 2.626 & $1.321-5.586$ & $0.005^{\star}$ \\
\hline Lymphatic metastasis & 1.324 & $0.798-2.676$ & 0.562 & & & \\
\hline Margins & 3.146 & $1.425-10.168$ & $<0.001^{\star}$ & 3.014 & $1.526-9.862$ & $0.012^{*}$ \\
\hline Recurrence & 1.625 & $0.964-3.122$ & 0.425 & & & \\
\hline T-cadherin methylation & 4.628 & $1.262-12.348$ & $<0.001^{\star}$ & 4.201 & $1.562-10.038$ & $<0.001^{*}$ \\
\hline
\end{tabular}

Note: ${ }^{*}$ p-value $<0.05$ was considered statistically significant

\section{A}

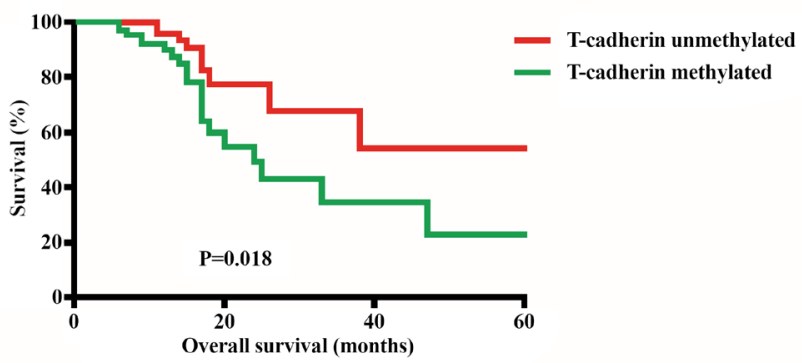

B

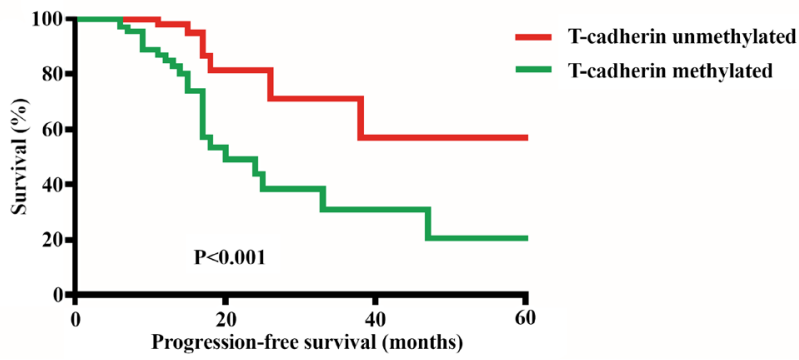

Figure 3. Kaplan-Meier curves for oral squamous cell carcinoma patients. Kaplan-Meier curves of overall survival (A) and disease-free survival (B) according to the methylation status of T-cadherin.

an important potential molecular marker for the diagnoses, effective monitoring, and prognosis of OSCC patients. Detection of DNA methylation in sera has the advantage of being easy to acquire, non-invasive, easy to store, and accessible to repeated acquisition [23]. In the present study, MSP and BSP were used to examine the methylation status of T-cadherin in the sera and tissues of OSCC patients and controls respectively, so as to explore the clinical significance and prognostic value of T-cadherin methylation in OSCC.

The current study demonstrated that the methylation status of T-cadherin in OSCC tissues was in accordance with that in serum, the results indicate that the methylation of T-cadherin in sera was derived from OSCC tissues.
Moreover, methylation of T-cadherin was associated with advanced stage and lymph node metastasis, which are all risk factors for tumor progression and poor prognosis. The results suggest, then, that methylation of T-cadherin in the sera of OSCC patients is closely correlated with the malignant behavior of the tumor and can be used as a valuable marker for these patients. Moreover, accumulating studies have confirmed that hypermethylation of $\mathrm{CpG}$ islands in the promoter region of the tumor suppressor gene can eventually lead to tumorigenesis, our previous study demonstrated that T-cadherin is a tumor suppressor gene, and its low expression is correlated with advanced clinical stage and PFS of OSCC and the overexpression of T-cadherin can inhibit proliferation of OSCC through the PI3K/AKT/mTOR pathway [14]. Thus, we speculate that loss of T-cadherin by promoter methylation promotes tumor progression and lymph node metastasis through activating the PI3K/AKT/mTOR pathway, finally leads to poor prognosis of OSCC patients, the exact mechanism needs further study to uncover this.

Recurrence is one of the main reasons for treatment failure in OSCC patients, $60 \%$ of whom are at risk for local recurrence, the patients who have recurrence also tend to have higher mortality rates and less favorable prognosis, with a median survival rate of only 10 months [24]. The removal of T-cadherin as a result of promoter methylation has been reported to be an important determinant of recurrence in various human cancers $[15,25]$. Our study discovered that methylation of T-cadherin in the sera of OSCC patients occurred more frequently in patients who had recurrent OSCC, and this suggests that T-cadherin methylation might be able to be used as a biomarker for predicting recurrence in OSCC patients [15].

The five-year survival rate of OSCC patients remains very low, and so far, there are few molecular markers that can effectively predict the prognosis in such patients [7]. Therefore, searching for molecular markers that can effectively predict the prognosis of OSCC patients is of great importance for the treatment of cancer. Our results found that the survival outcome of OSCC patients with methylated T-cadherin was worse, which suggests that methylation of T-cadherin in the sera of OSCC patients is closely correlated with a less favor- 
able prognosis. Multivariate analysis suggests that methylation of T-cadherin is an independent factor for the OS and PFS rates of patients with OSCC. These results are similar to the findings for bladder and renal cancer $[15,26]$. The data suggest that methylation of T-cadherin in the sera prior to treatment can be an independent predictor for the prognosis of OSCC patients.

In conclusion, the present study demonstrated that methylation of T-cadherin occurred frequently in the sera of patients with OSCC before treatment and was associated with advanced stage, positive lymph node metastases, tumor recurrence, and poor prognosis. The current study suggests that methylation of T-cadherin in the sera should be determined before treatment and that, for the patients with methylated T-cadherin in sera, enhanced follow-up and effective targeted therapy should be performed to prevent tumor relapse and obtain a better prognosis.

Acknowledgments: This study was supported by the Health Department of Sichuan Province (grant/award number: 20PJ110, 20PJ116); the Sichuan Provincial Cadre Health Research Project (grant/award number: 2019-802); Research Project of Sichuan Administration of Traditional Chinese Medicine (2020JC0114); Youth Scientific research Project of the Third Affiliated Hospital of Guangzhou Medical University (grant/award number: 2018Q4). The authors have no other relevant affiliations or financial involvement with any organization or entity with a financial interest in or financial conflict with the subject matter or materials discussed in the manuscript apart from those disclosed. No writing assistance was utilized in the production of this manuscript.

\section{References}

[1] BRAY F, FERLAY J, SOERJOMATARAM I, SIEGEL RL, TORRE LA et al. Global cancer statistics 2018: GLOBOCAN estimates of incidence and mortality worldwide for 36 cancers in 185 countries. CA Cancer J Clin 2018; 68: 394-424. https://doi.org/10.3322/caac.21492

[2] COLEVAS AD, YOM SS, PFISTER DG, SPENCER S, ADELSTEINET D et al. NCCN guidelines insights: head and neck cancers, Version 1.2018. J Compr Canc Netw 2018; 16: 479490. https://doi.org/10.6004/jnccn.2018.0026

[3] ARGIRIS A, KARAMOUZIS MV, RABEN D, FERRIS RL. Head and neck cancer. Lancet 2008; 371: 1695-1709. https:// doi.org/10.1016/S0140-6736(08)60728-X

[4] VERMORKEN JB, MESIA R, RIVERA F, REMENAR E, KAWECKI A et al. Platinum-based chemotherapy plus cetuximab in head and neck cancer. N Engl J Med 2008; 359: 1116-1127. https://doi.org/10.1056/NEJMoa0802656

[5] BAUML J, SEIWERT TY, PFISTER DG, WORDEN F, LIU SV et al. Pembrolizumab for platinum- and cetuximab-refractory head and neck Cancer: results from a single-arm, phase II study. J Clin Oncol 2017; 35: 1542-1549. https://doi. org/10.1200/JCO.2016.70.1524
[6] FERRIS RL, BLUMENSCHEIN GJ, FAYETTE J, GUIGAY J, COLEVAS AD et al. Nivolumab for recurrent squamous-cell carcinoma of the head and neck. N Engl J Med 2016; 375: 1856-1867. https://doi.org/10.1056/NEJMoa1602252

[7] CHAI AWY, LIM KP, CHEONG SC. Translational genomics and recent advances in oral squamous cell carcinoma. Semin Cancer Biol 2020; 61: 71-83. https://doi.org/10.1016/j.semcancer.2019.09.011

[8] WANG Z, LING S, RETTIG E, SOBEL R, TANET $M$ et al. Epigenetic screening of salivary gland mucoepidermoid carcinoma identifies hypomethylation of CLIC3 as a common alteration. Oral Oncol 2015; 51: 1120-1125. https://doi. org/10.1016/j.oraloncology.2015.09.010

[9] DAWSON MA, KOUZARIDES T. Cancer epigenetics: From mechanism to therapy. Cell 2012; 150: 12-27. https://doi. org/10.1016/j.cell.2012.06.013

[10] WARTON K, MAHON KL, SAMIMI G. Methylated circulating tumor DNA in blood: Power in cancer prognosis and response. Endocr Relat Cancer 2016; 23: R157-171. https:// doi.org/10.1530/ERC-15-0369

[11] JITHESH PV, RISK JM, SCHACHE AG, DHANDA J, LANE B et al. The epigenetic landscape of oral squamous cell carcinoma. Br J Cancer 2013; 108: 370-379. https://doi. org/10.1038/bjc. 2012.568

[12] ABUDUKADEER A, BAKRY R, GOEBEL G, MUTZ-DEHBALAIE I, WIDSCHWENDTER A et al. Clinical Relevance of CDH1 and CDH13 DNA-Methylation in Serum of Cervical Cancer Patients. Int J Mol Sci 2012; 13: 8353-8363. https://doi.org/10.3390/ijms13078353

[13] DUAN XS, LU J, GE ZH, XING EH, LU HT et al. Effects of Tcadherin expression on B16F10 melanoma cells. Oncol Lett 2013; 5: 1205-1210. https://doi.org/10.3892/ol.2013.1164

[14] WANG QJ, ZHANG XQ, SONG XY, ZHANG L et al. Overexpression of T-cadherin inhibits the proliferation of oral squamous cell carcinoma through the PI3K/AKT/mTOR intracellular signalling pathway. Arch Oral Biol 2018; 96: 74-79. https://doi.org/10.1016/j.archoralbio.2018.08.018

[15] LIN YL, SUN G, LIU XQ, LI WP, MA JG. Clinical Significance of CDH13 Promoter Methylation in Serum Samples from Patients with Bladder Transitional Cell Carcinoma. J Int Med Res 2011; 39: 179-186. https://doi. org/10.1177/147323001103900119

[16] WANG L, LIN YL, LI B, WANG YZ, LI WP, et al. Aberrant promoter methylation of the cadherin 13 gene in serum and its relationship with clinicopathological features of prostate cancer. J Int Med Res 2014; 42: 1085-1092. https://doi. org/10.1177/0300060514540631

[17] HE X, LI JP, LIU XH, et al. Prognostic value of C-reactive protein/albumin ratio in predicting overall survival of Chinese cervical cancer patients overall survival: comparison among various inflammation based factors. J Cancer 2018; 9: 1877-1884. https://doi.org/10.7150/jca.23320

[18] LIN YL, LI YL, MA JG. Aberrant Promoter Methylation of Protocadherin8 (PCDH8) in Serum is a Potential Prognostic Marker for Low Gleason Score Prostate Cancer. Med Sci Monit 2017; 23: 4895-4900. https://doi.org/10.12659/ msm.904366 
[19] WANG WW, CHEN GY, WANG B et al. Long non-coding RNA BZRAP1-AS1 silencing suppresses tumor angiogenesis in hepatocellular carcinoma by mediating THBS1 methylation. J Transl Med 2019; 17: 421. https://doi.org/10.1186/ s12967-019-02145-6

[20] KIM YK, KIM WJ. Epigenetic markers as promising prognosticators for bladder cancer. Int J Urol 2009; 16: 17-22. https://doi.org/10.1111/j.1442-2042.2008.02143.x

[21] CHAO C, CHI M, PRECIADO M, BLACK MH. Methylation markers for prostate cancer prognosis: a systematic review. Cancer Causes Control 2013; 24: 1615-1641. https://doi. org/10.1007/s10552-013-0249-2

[22] SWARUP V, RAJESWARI MR. Circulating (cell-free) nucleic acids--a promising, non-invasive tool for early detection of several human diseases. FEBS Lett 2007; 581:795-799. https://doi.org/10.1016/j.febslet.2007.01.051
[23] HOW KA, NIELSEN HM, TOST J. DNA methylation based biomarkers: Practical considerations and applications. Biochimie 2012; 94:2314-2337. https://doi.org/10.1016/j.biochi.2012.07.014

[24] SACCO GA, COHEN EE. Current treatment options for recurrent or metastatic head and neck squamous cell carcinoma. J Clin Oncol 2015; 33: 3305-3313. https://doi. org/10.1200/JCO.2015.62.0963

[25] ANDREEVA AV, KUTUZOV MA. Cadherin 13 in cancer. Genes Chromosomes Cancer 2010; 49: 775-790. https://doi. org/10.1002/gcc.20787

[26] WANG L, LIN YL, LI B, WANG YZ, LI WP, et al. Aberrant promoter methylation of the cadherin 13 gene in serum and its relationship with clinicopathological features of prostate cancer. J Int Med Res 2014; 42: 1085-1092. https://doi. org/10.1177/0300060514540631 